\title{
MM3 Potential energy surfaces of $\beta$-4-linked mannobiose and mannotriose at different dielectric constants*
}

\author{
Carlos A Stortz \\ Departamento de Química Orgánica-CIHIDECAR, Facultad de Ciencias Exactas y Naturales, \\ UBA. Ciudad Universitaria, Pab. 2, 1428 Buenos Aires, Argentina \\ E-mail:stortz@qo.fcen.uba.ar
}

\section{Dedicated to Dr. R. M. Lederkremer on the occasion of her $\mathbf{7 0}^{\text {th }}$ birthday}

\begin{abstract}
The adiabatic potential energy surface (PES) of $\beta$-4-linked mannobiose was obtained using the MM3 force field at $\varepsilon=3$ and $\varepsilon=80$, and plotted as contour maps and as 2D graphs representing the energy vs. the $\psi$ angle. The surfaces of the corresponding trisaccharide were also obtained and represented by a single 3D contour map for which the energy is plotted against the two $\psi$ glycosidic angles. The PES of the disaccharide contains a low-energy well comprising two different minima, and three more minima in different locations. No major change was observed by changing the dielectric constant. For the trisaccharide, four main minima were observed, located within one minimum-energy region. The minima have a geometry close to that experimentally obtained for mannobiose, mannotriose and mannan I in solid state, but differ from that expected in aqueous solutions. The flexibility of the glycosidic linkage increases at higher dielectric constant, whereas it decreases for the linkage closer to the reducing end when passing from the di- to the trisaccharide.
\end{abstract}

Keywords: Trisaccharides, mannan, mannobiose, conformational analysis, MM3, potential energy surface

\section{Introduction}

In order to understand the industrial and biological functions of carbohydrates, a deep knowledge of their shapes is very important. The conformational analysis of carbohydrates is usually carried out by both experimental and modeling techniques. ${ }^{1}$ Disaccharides are modeled by generating a Ramachandran-like 3D contour plot relating the potential energy of the molecules and the glycosidic angles $\phi$ and $\psi \cdot{ }^{1-3}$ Originally made with rigid residues, many of those maps were performed lately by flexible-residue analysis in a systematic way, ${ }^{4-7}$ especially after the 
appearance of the force-field MM3,8,9 considered very reliable for carbohydrates given its considerations to hydrogen-bond forces, anomeric and exo-anomeric effects. As the glycosidic angle $\phi$ takes a value more or less fixed it can be allowed to relax, and the energy can be related directly to $\psi$ in a $x y$ plot. ${ }^{10,11}$ Furthermore, the potential energy surfaces (PES) of many trisaccharides were presented as contour maps in which the potential energy is plotted as a function of both $\psi$ angles. ${ }^{12-15}$ In most cases, very little influence of one linkage over the other is found to occur, ${ }^{12-15}$ but in some trisaccharides a hydrogen bond between the first and third units alters the shape of the PES expected from putting both disaccharide maps together. ${ }^{13,14}$

Cellulose, the best-known fibrillar polysaccharide is composed of $\beta$-1,4-linked D-glucose units. In some plant tissues, the D-glucose units of cellulose are partly replaced by D-mannose units, to form glucomannans. Furthermore, either in cell walls or in seed endosperms, linear skeletons of $\beta$-4-linked D-mannans are found, variably substituted with $\alpha$-D-galactosyl units on C-6. They may range from practically unsubstituted mannans (the name "mannan" is kept for those with less $15 \%$ substitution) to heavily substituted galactomannans. ${ }^{16}$ The gelling properties of these polysaccharides render industrial value. Several modeling attempts for mannan and galactomannan oligomers were made: mannobiose was studied in solution, ${ }^{17,18}$ and a strong influence of the solvent was found to occur. The effect of galactosyl substitution in galactomannan chains was also studied. ${ }^{19,20}$ With the aid of MM3 (92), a study of mannobiose and several galactosyl-substituted mannobioses was made at high dielectric constant. ${ }^{20}$ By X-ray crystallography, both the di- and the trisaccharide were studied. ${ }^{21,22}$ Herein, in connection with the appearance of oligo mannans in a galactomannan-rich endosperm, ${ }^{23}$ the potential energy surfaces of $\beta$-4-linked mannobiose and mannotriose are presented at low and high dielectric constants.
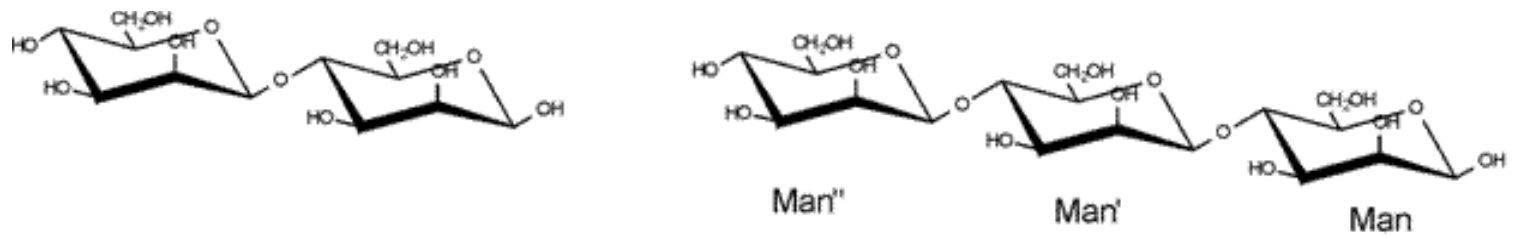

Structure 1. The di- and trisaccharide studied in this work.

\section{Results and Discussion}

The MM3 adiabatic potential energy surfaces (PES) for $\beta$-mannobiose (Structure 1) at $\varepsilon=3$ and 80 , depicted as energy plots related to the glycosidic angle $\psi^{10}$ (i.e. with the $\phi$ angles relaxed to their minimum-energy position in the main trough) are shown on Fig. 1, while the corresponding 3D contour maps for the area surrounding the major minima are shown on Fig. 2. Table 1 shows the geometrical and energy characteristics of the lower-energy minima in each region. Within the main trough of angle $\phi$, three minima are observed, with very small energy differences between 
those labeled as $\mathbf{A}$ and $\mathbf{B}$, and a very small influence of the dielectric constant on their energy differences (Table 1). A third minimum (C) appears in the same $\phi$ area but with a different $\psi$ value. Two other minima appear (Table 1). One of them (B') carries a $\psi$ angle similar to that of $\mathbf{B}$, but a different $\phi$ angle, not promoted by the exo-anomeric effect. ${ }^{7}$ Its appearance is erratic in $\beta$-linked disaccharides: ${ }^{7,11}$ it is observed in laminaribiose and sophorose at low dielectric constants, but not for cellobiose. 7 The other minimum (D) corresponds to the "side of the map" region, with $\phi^{\mathrm{H}}$ close to $180^{\circ}$, which also exhibits exo-anomeric effect. These three secondary minima are more stable at $\varepsilon=80$ than at $\varepsilon=3$, in contrast with other MM3 calculations which show a decrease of the stability of minimum $\mathbf{D}$ with an increase of the dielectric constant. ${ }^{7}$

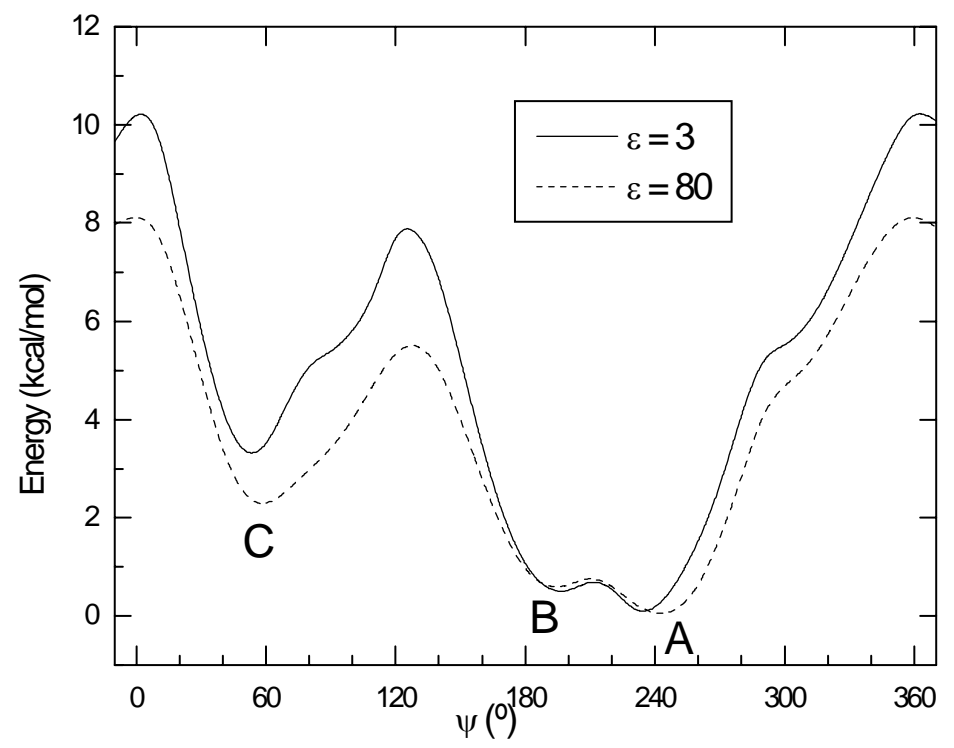

Figure 1. MM3 relaxed surface (2D plot) for $\beta$-mannobiose at $\varepsilon=3$ and $\varepsilon=80$.

The MM3 PES of the trisaccharide $\beta$-mannotriose (Str. Block 1) determined as a contour map relating the energy to both $\psi$ glycosidic angles at $\varepsilon=3$ and $\varepsilon=80$ is shown in Fig. 3, calculated around the region A-B of low energy (both $\psi$ angles from 160 to $280^{\circ}$ ). The geometrical and energy data on the main minima obtained in each region at both dielectric constants are shown on Table 2. The minima are named by a two-letter code according to the minimum energy region (A or B) of the Man" $\rightarrow$ Man' linkage (first letter) and the Man' $\rightarrow$ Man linkage (second letter). ${ }^{12-15}$ Table 3 shows the flexibility measurements carried out using the data obtained for the di- and trisaccharides under study. 

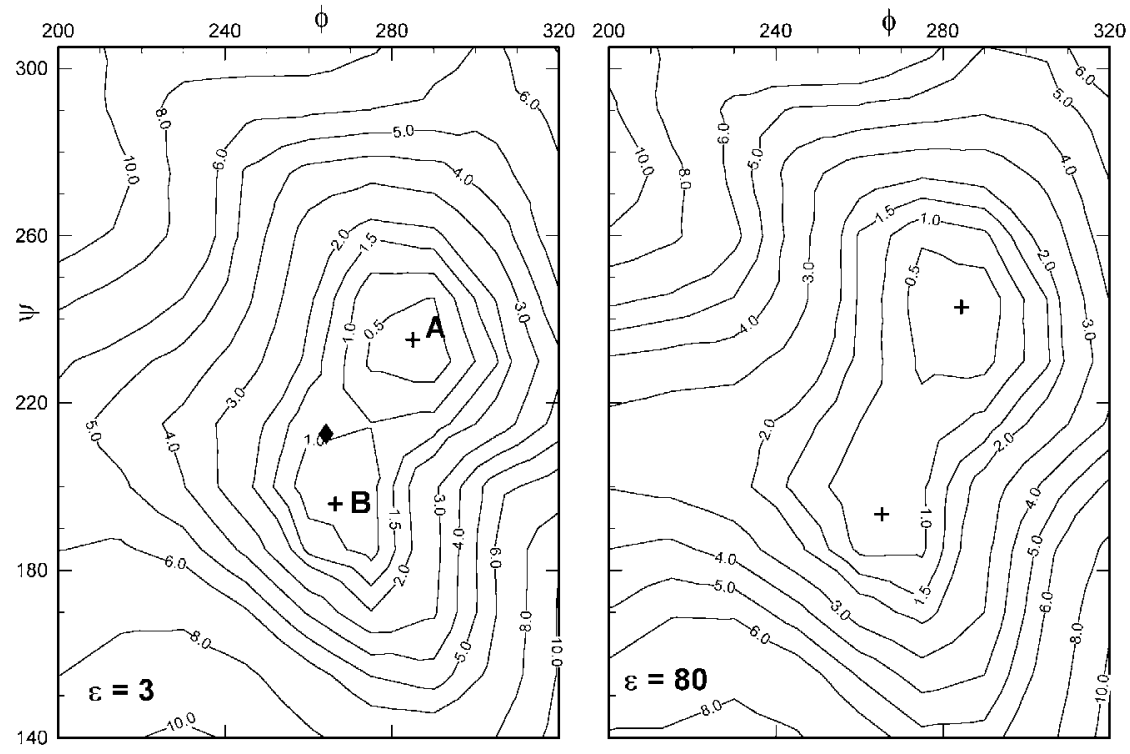

Figure 2. MM3 adiabatic conformational maps (3D, main area) of $\beta$-mannobiose at $\varepsilon=3$ and $\varepsilon=80$. Iso-energy contour lines are graduated in the indicated increments above the global minimum. The symbols indicate: $(+)$ MM3 minima; $(\bullet)$ crystal structure of mannobiose. ${ }^{21}$

Table 1. Torsion angles $\left({ }^{\circ}\right)$, relative steric and free energies $(\mathrm{kcal} / \mathrm{mol})$ and exocyclic angles for the minimum-energy conformations obtained for $\beta$-mannobiose at $\varepsilon=3$ and $\varepsilon=80$ with the MM3 force-field. Selected data for the main transition states (TS) is also included.

\begin{tabular}{|c|c|c|c|c|c|c|}
\hline & \multirow[t]{2}{*}{$\phi, \psi$} & \multirow[t]{2}{*}{$\phi^{\mathrm{H}}, \psi^{\mathrm{H}}$} & \multirow[t]{2}{*}{$\mathrm{E}_{\text {rel }}$} & \multirow[t]{2}{*}{$\mathrm{G}_{\text {rel }}$} & \multicolumn{2}{|c|}{ Exocyclic torsion angles ${ }^{a}$} \\
\hline & & & & & $\chi_{2}^{\prime} \chi_{3}^{\prime} \chi_{4}^{\prime} \omega^{\prime} \chi_{6}^{\prime}$ & $\chi_{1} \chi_{2} \chi_{3} \omega_{\omega} \chi_{6}$ \\
\hline \multicolumn{7}{|l|}{$(\varepsilon=3)$} \\
\hline A & 285,235 & $47,-5$ & 0.00 & 0.00 & gTG gG & TGG gG \\
\hline B & 267,195 & $29,-47$ & 0.46 & 0.38 & gTG gT & TGG gG \\
\hline $\mathbf{B}^{\prime}$ & 184,199 & $-59,-42$ & 5.15 & 4.83 & gTG gG & TGS Gg \\
\hline $\mathbf{C}$ & 275,53 & 38,172 & 3.14 & 4.03 & gTG gG & TGG gG \\
\hline $\mathbf{D}$ & 39,245 & 157,7 & 7.38 & 8.71 & GGg $\mathrm{gG}$ & TGG Gg \\
\hline $\mathrm{TS} \mathbf{A} \leftrightarrow \mathbf{B}$ & 272,220 & $34,-21$ & 0.97 & & & \\
\hline \multicolumn{7}{|l|}{$(\varepsilon=80)$} \\
\hline A & 284,243 & 46,4 & 0.00 & 0.00 & ggG gT & gGG gT \\
\hline B & 265,193 & $27,-49$ & 0.53 & 0.48 & ggG gT & gGg gT \\
\hline $\mathbf{B}^{\prime}$ & 182,195 & $-61,-47$ & 3.33 & nd & GgG gT & gGg gT \\
\hline $\mathbf{C}$ & 271,57 & 34,175 & 2.08 & 2.83 & ggG gT & ggG gT \\
\hline D & 58,237 & $175,-3$ & 5.97 & 6.88 & GgG gT & ggg gT \\
\hline $\mathrm{TS} \mathbf{A} \leftrightarrow \mathbf{B}$ & 273,215 & $35,-26$ & 0.91 & & & \\
\hline
\end{tabular}

aFor nomenclature, see Methods section. The geometry indicated corresponds to that of each steric energy minimum. 
Table 2. Torsion angles $\left({ }^{\circ}\right)$, relative steric energies $(\mathrm{kcal} / \mathrm{mol})$ and exocyclic angles for the main minimum-energy conformations obtained for $\beta$-mannotriose at $\varepsilon=3$ and $\varepsilon=80$ with the MM3 force-field. Selected data for the main transition states (TS) is also included.

\begin{tabular}{|c|c|c|c|c|c|c|c|}
\hline & $\begin{array}{c}\phi_{\text {Man" } \rightarrow \text { Man', }}, \\
\psi \text { Man" } \rightarrow \text { Man' } \\
\left(\phi^{\mathrm{H}}, \psi^{\mathrm{H}}\right)\end{array}$ & $\begin{array}{c}\phi \text { Man' } \rightarrow \text { Man }, \\
\psi \text { Man' } \rightarrow \text { Man } \\
\quad\left(\phi^{\mathrm{H}}, \psi^{\mathrm{H}}\right)\end{array}$ & $\mathrm{E}_{\mathrm{rel}}$ & $\mathrm{G}_{\mathrm{rel}}$ & Exocyc & $\mathrm{c}$ torsion & angles ${ }^{a}$ \\
\hline \multicolumn{8}{|l|}{$(\varepsilon=3)$} \\
\hline AA & $286,235(47,-5)$ & $286,236(48,-3)$ & 0.00 & 0.00 & gTG gG & GG gG & TGG Gg \\
\hline $\mathbf{A B}$ & 286, $234(47,-5)$ & $267,197(29,-46)$ & 1.33 & 0.62 & gTG gG & GG gT & TGG gG \\
\hline BA & $268,194(30,-48)$ & $287,235(49,-5)$ & 0.39 & 0.21 & gTG gT & GG gG & TGG gG \\
\hline BB & $270,195(32,-47)$ & $267,195(30,-47)$ & 1.67 & 0.98 & gTG gG & $\mathrm{gT}$ gT & TGG gG \\
\hline $\begin{array}{c}\mathrm{TS} \\
\mathbf{A A} \leftrightarrow \mathbf{A B}\end{array}$ & $286,234(46,-5)$ & $274,212(36,-29)$ & 1.58 & & & & \\
\hline $\mathbf{A A} \leftrightarrow \mathbf{B A}$ & $273,221(35,-21)$ & $287,235(48,-5)$ & 0.99 & & & & \\
\hline $\mathrm{AB} \leftrightarrow \mathrm{BB}$ & $272,217(34,-25)$ & $267,198(30,-45)$ & 2.16 & & & & \\
\hline $\mathrm{BA} \leftrightarrow \mathrm{BB}$ & $269,193(31,-49)$ & $273,212(35,-29)$ & 1.94 & & & & \\
\hline \multicolumn{8}{|l|}{$(\varepsilon=80)$} \\
\hline AA & $284,244(46,5)$ & $284,243(46,4)$ & 0.00 & 0.00 & $\operatorname{ggG} g \mathrm{~T}$ & $\mathrm{gG} g \mathrm{~T}$ & gGG gT \\
\hline $\mathbf{A B}$ & $284,243(46,4)$ & $265,193(27,-49)$ & 0.54 & 0.46 & ggG gT & $\mathrm{gGgT}$ & gGg gT \\
\hline BA & $265,193(27,-49)$ & $284,243(46,4)$ & 0.49 & 0.46 & ggG gT & gg gT & gGG gT \\
\hline BB & $265,193(27,-49)$ & $265,193(27,-49)$ & 1.03 & 0.92 & $\operatorname{ggG} g \mathrm{~T}$ & gg gT & gGg gT \\
\hline $\begin{array}{c}\mathrm{TS} \\
\mathbf{A A} \leftrightarrow \mathbf{A B}\end{array}$ & $284,243(46,4)$ & $269,210(31,-32)$ & 0.73 & & & & \\
\hline $\mathbf{A A} \leftrightarrow \mathbf{B A}$ & $273,215(36,-26)$ & $284,243(46,4)$ & 0.88 & & & & \\
\hline $\mathbf{A B} \leftrightarrow \mathbf{B B}$ & $269,210(31,-32)$ & $265,193(27,-49)$ & 1.31 & & & & \\
\hline $\mathrm{BA} \leftrightarrow \mathrm{BB}$ & $266,192(29,-50)$ & $269,206(31,-36)$ & 1.26 & & & & \\
\hline
\end{tabular}

aThe angles are given in the order $\chi_{2}$ " $\chi_{3} " \chi_{4} " \omega^{\prime \prime} \chi_{6}$ " $\chi_{2}^{\prime} \chi_{3}{ }^{\prime} \omega^{\prime} \chi_{6}{ }^{\prime} \chi_{1} \chi_{2} \chi_{3} \quad \omega_{6} \chi_{6}$.For nomenclature of the angles, see Methods section. The geometry indicated corresponds to that of each steric energy minimum.

Flexible-residue molecular modeling was systematically used for many different disaccharides, ${ }^{1-7}$ especially after the appearance of $M M 33^{8,9}$ a force-field with good parameterization for carbohydrates. Those studies were recently extended to different trisaccharides, ${ }^{12-15}$ in an attempt to achieve a better knowledge of the conformational features of polysaccharides. For $\beta$-mannobiose at $\varepsilon=3$, present calculations show the typical pattern (Figs. 1 and 2, Table 1), with very little energy difference between the minima labeled as $\mathbf{A}$ and $\mathbf{B}$. These results are very similar to those observed for $\beta$-cellobiose at $\varepsilon=4$ with the same force field, ${ }^{12}$ 
especially with respect to the geometry of the minima. However, the present results show $\mathbf{A}$ as the global minimum $(0.46 \mathrm{kcal} / \mathrm{mol}$ below minimum B), whereas for cellobiose the minimum $\mathbf{B}$ was slightly more stable $(0.10 \mathrm{kcal} / \mathrm{mol})$. Besides, a minimum in the non-exo-anomeric B' area (around $\phi, \psi 180^{\circ},-160^{\circ}$ ) is observed for $\beta$-mannobiose (Table 1), whereas for $\beta$-cellobiose this area was shallow, but did not have a local minimum. ${ }^{12}$ When the calculations were repeated at a dielectric constant of 80 , where the electrostatic and hydrogen bond interactions are almost suppressed, ${ }^{3}$ no major difference is encountered for the energy difference between both minima This result is probably due to the fact that the hydrogen bonding arrangements are very similar in minima A and B, occurring mainly between $\mathrm{H}(\mathrm{O} 3)$ and O5' (Table 5). This interaction is slightly weakened in B, but this minimum also shows another hydrogen bond between $\mathrm{H}(\mathrm{O} 3)$ and O6', among other interactions present in both minima. The crystal structure reported for $\alpha$ mannobiose $^{21}$ shows a $\phi, \psi$ of $264^{\circ}, 212^{\circ}$, in-between minima $\mathbf{A}$ and $\mathbf{B}$, but closer to the latter. The hydrogen bond interaction between $\mathrm{H}(\mathrm{O} 3)$ and $\mathrm{O}^{\prime}$ ' in this crystal structure is probably stronger than that observed in the calculated A or $\mathbf{B}$ minima, as assumed from the shorter distance between both atoms $(2.03 \AA)$. The crystal structure of the disaccharide exhibits a different orientation of both hydroxymethyl groups: that of the non-reducing moiety appears as the GT rotamer ( $\mathrm{g}$ in our nomenclature), whereas that of the reducing moiety appears as the GG rotamer $(\mathrm{G}){ }^{21}$ Our calculations indicate that such combination of rotamers is preferential for minima B' and D, whereas the low-energy minima A, B and $\mathbf{C}$ are GT-GT rotamers (Table 1). However, the expected GT-GG conformer in the A region appears with an energy only 0.07 $\mathrm{kcal} / \mathrm{mol}$ higher than that of the global minimum. Fiber diffraction analysis of mannan I indicate that the backbone glycosidic angles appear well inside our $\mathbf{B}$ region: $\phi, \psi=279^{\circ}, 199^{\circ}$ according to electron diffraction analysis, ${ }^{24}$ and $270^{\circ}, 211^{\circ}$ according to X-ray diffraction analysis. ${ }^{25}$ Both determinations indicated that the hydroxymethyl groups were at their GT orientations, and the presence of inter-residual intra-chain hydrogen bonds between $\mathrm{H}(\mathrm{O} 3)$ and $\mathrm{O} 5$, and between $\mathrm{H}(\mathrm{O} 6)$ and $\mathrm{O} 3$.

Previous calculations with MM3(92) at $\varepsilon=80$ showed a qualitative pattern similar to that presented here. ${ }^{20}$ However, in that work instead of minimum B', a different minimum within the main $\phi$ trough was reported to occur. MM2CARB calculations have also conducted to a similar qualitative pattern, ${ }^{18}$ with the same five minima as the present paper, but lower relative energies for the lateral minima B' and $\mathbf{D}$, as expected from a method which gave less weight to the exoanomeric effect than MM3. Former PCILO (a semiempirical method) calculations gave rise to a different pattern: ${ }^{17}$ seven minima were encountered, five of which match those of the present calculation. However, the global minimum corresponded to one not found in other calculations. Furthermore, the proportion of this minimum was increased when the polarity of an emulated solvent was raised. ${ }^{17}$ Anyway, the energies of minima $\mathbf{A}$ and $\mathbf{B}$ were only slightly higher than that of the global minimum. On the other hand, newer PCILO calculations which included a modeling of water solvation indicated the presence of three main minima, ${ }^{19}$ the global one corresponding to the $\mathbf{D}$ region, followed by another in a region not identified by other methods, while the third was close to our B region (the solid-state conformation). The authors of the latter 
paper have determined experimentally the coupling constants, optical rotation, and NOESY spectrum of mannobiose and found an excellent agreement with their calculated Boltzmannaveraged populations. ${ }^{19}$ The conclusion reached is the presence of major amounts of $\mathbf{D}$ conformers in solution, as opposed to the exclusive occurrence of A-B conformers in the solid state. Although the experimental ${ }^{3} \mathrm{~J} \psi$ and linkage rotation values can also be explained in terms of different $\mathbf{A} / \mathbf{B}$ equilibria, the ${ }^{3} \mathrm{~J} \phi$ value and the $\mathrm{r}_{\mathrm{H} 1}$ '-H4 deduced from the NOESY spectrum seem to indicate that $\mathbf{D}$ conformers ("side of the map" minima ${ }^{7}$ ) can actually be preponderant in solution. However, most of the molecular modeling packages are unable to detect this minimum as the global one, probably due to their difficulties to handle a polar environment. MM3 shows a decrease of its energy at $\varepsilon=80$ (Table 1), although it is still too high. Modeling at $\varepsilon=80$ was found to give better coincidence with experimental data in water than that at lower dielectric constants in a couple of examples. ${ }^{3,15}$ However, the absence of hydrogen bonding and electrostatic forces at such high dielectric constant may be inducing deformations in the calculations not predicted by its parameterization, expected to work at lower dielectric constants.

Modeling of the corresponding trisaccharide leads to four main minima (AA, AB, BA and BB) with very similar energies (Table 2, Fig. 3), and a map resembling closely that observed for $\beta$-cellotriose. ${ }^{12}$ AA appears as the global minimum (Fig. 4), tightly followed by BA. The order is maintained, but the energies appear to be nearer when calculating free energies (Table 2) or when the calculations were repeated at $\varepsilon=80$. It has already been observed that both calculations yield similar results. ${ }^{26}$ As occurs with cellotriose and other trisaccharides, ${ }^{12-15}$ the surfaces are those expected from disaccharides maps, i.e. no special interaction between the first and third unit appears altering the expected PES, as happens for some trisaccharides. ${ }^{13,14}$ The orientation of the exocyclic groups is similar to that occurring for the disaccharide (cf Tables 1 and 2), being those of the middle unit similar to those at the reducing end. However, the global minimum shows a GT hydroxymethyl orientation of the non-reducing and middle units, but a GG orientation of the reducing end, as observed in the crystal structure of mannobiose. ${ }^{21}$ Anyway, the energy of the conformer carrying the three moieties as their GT rotamers is only 0.05 $\mathrm{kcal} / \mathrm{mol}$ above. The crystal structure determined for a hydrate of mannotriose 22 shows precisely this orientation of the hydroxymethyl groups and a combination of $\phi, \psi$ angles located very close to our AB minimum (Figure 3). 

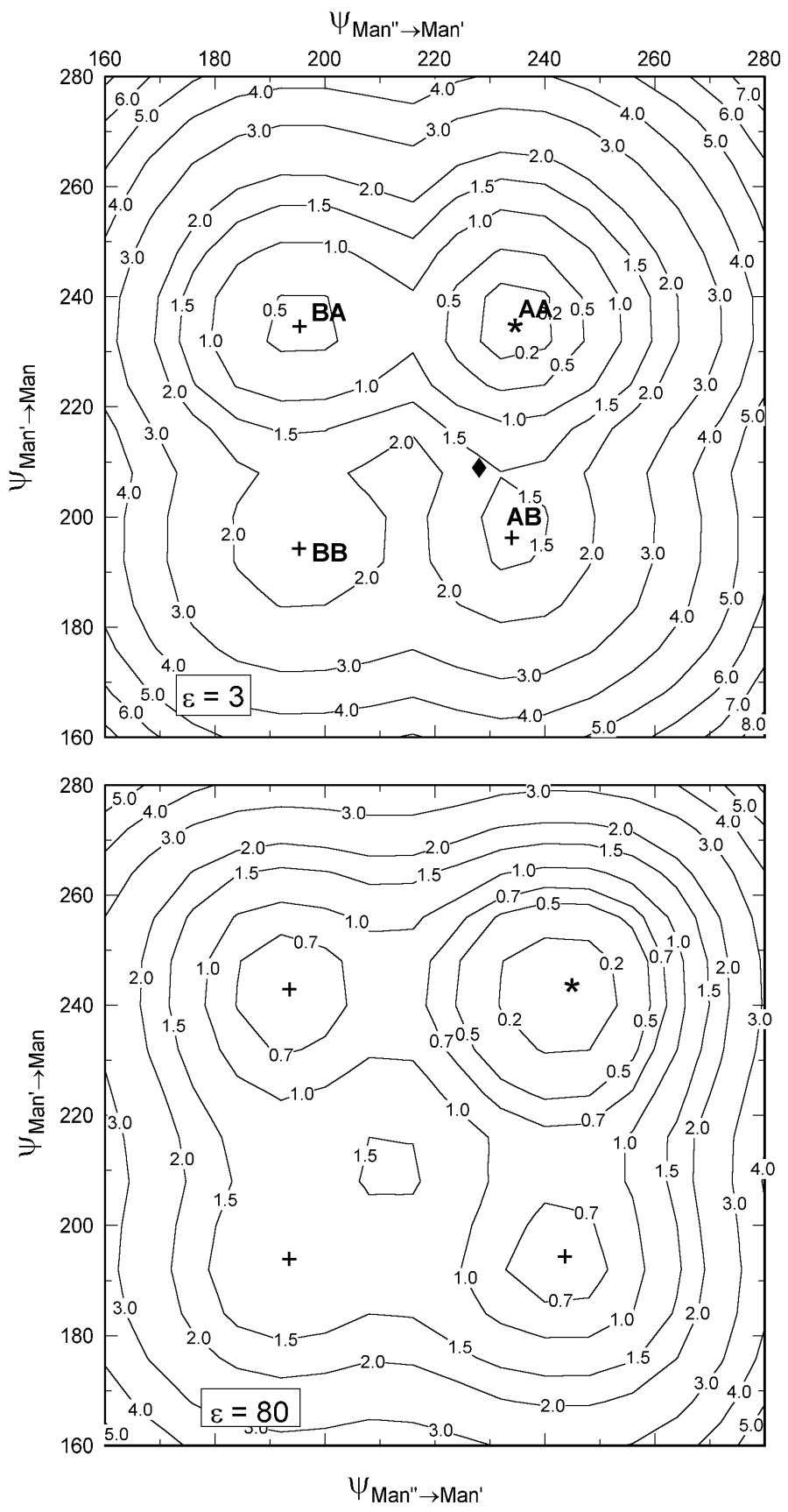

Figure 3. MM3 adiabatic conformational maps (energy vs. $\psi$ Man" $\rightarrow$ Man', $\psi$ Man' $\rightarrow$ Man) of $\beta$ mannotriose at $\varepsilon=3$ and $\varepsilon=80$ in the lower energy-region. Iso-energy contour lines are graduated in the indicated increments above the global minimum. The symbols indicate: (*) MM3 global minima; $(+)$ MM3 local minima; $(\diamond)$ crystal structure of mannotriose. $^{22}$

The flexibility of the glycosidic linkage has been measured in terms of a partition function, ${ }^{11,12,26}$ or probability volume. ${ }^{27}$ This parameter is very sensitive to the regions of the 
PES with very low energy, and thus, it is highly influenced by the entropy of the global minimum. Another parameter used for carbohydrates is the "absolute flexibility"11,26,28,29 which gives an indication of the ease of interconversion between the lower energy minima, being thus very sensitive to the height of the lower potential barriers. It has been shown that in trisaccharides, the individual flexibility of each glycosidic linkage can be estimated. ${ }^{12}$ The partition function for $\beta$-mannobiose at $\varepsilon=3$ is $890 \mathrm{deg}^{2}$ (Table 3). This value is slightly lower than that encountered by the all-equatorial cellobiose, ${ }^{12}$ and other disaccharides like maltose, ${ }^{12}$ sophorose, kojibiose ${ }^{14}$ and a fucobiose, ${ }^{15}$ but similar to other disaccharides. ${ }^{10}$ The flexibility increases by raising the dielectric constant (Table 3 ). This fact can also be deduced by observing Figure 2: the low energy surface is more extended when working at $\varepsilon=80$. The absolute flexibility measurements also give a value (0.0078) lower than those of other 4-linked disaccharides like maltose or cellobiose, ${ }^{12}$ but higher than those of other disaccharides ${ }^{14,15}$ which exhibit a larger distance between minima $\mathbf{A}$ and $\mathbf{B}$, and thus a higher potential barrier. As expected from its reduced potential barrier (Table 1), the absolute flexibility also increases at $\varepsilon=80$ (Table 3$)$.

Table 3. Corrected partition functions $q$ and absolute flexibilities $\Phi$ calculated for the compounds under study for $\beta$-mannobiose and $\beta$-mannotriose at $\varepsilon=3$ and $\varepsilon=80$.

\begin{tabular}{|c|c|c|c|c|c|c|}
\hline & & $\begin{array}{c}\mathrm{q}_{\psi} \\
(\mathrm{deg})\end{array}$ & $\begin{array}{c}\mathrm{q}_{\phi, \psi} \\
\left(\mathrm{deg}^{2}\right) \\
\end{array}$ & $\begin{array}{c}\Phi_{\phi, \psi} \\
\left(\mathrm{x} 10^{4}\right) \\
\end{array}$ & $\begin{array}{c}\Phi_{\psi} \\
\left(\mathrm{x} 10^{4}\right)\end{array}$ & $\Phi_{\phi, \psi} / \Phi_{\psi}$ \\
\hline \multicolumn{7}{|c|}{$\beta$-Mannobiose } \\
\hline$\varepsilon=3$ & & 39 & 890 & 78 & 99 & 0.79 \\
\hline \multirow[t]{2}{*}{$\varepsilon=80$} & & 47 & 1300 & 109 & 158 & 0.69 \\
\hline & $\begin{array}{c}\mathrm{q}_{\psi, \psi} \\
\left(\mathrm{deg}^{2}\right)\end{array}$ & $\begin{array}{c}\mathrm{q} \psi \\
(\mathrm{deg})\end{array}$ & $\begin{array}{c}\Phi_{\phi, \psi}, \phi, \psi \\
\left(\mathrm{x} 10^{4}\right)\end{array}$ & $\begin{array}{c}\Phi_{\psi, \psi} \\
\left(\mathrm{x} 10^{4}\right)\end{array}$ & $\begin{array}{c}\Phi_{\psi} \\
\left(\mathrm{x} 10^{4}\right)\end{array}$ & $\begin{array}{c}\Phi_{\phi, \psi, \phi, \psi} \\
/ \Phi_{\psi, \psi}\end{array}$ \\
\hline \multicolumn{7}{|c|}{$\beta$-Mannotriose } \\
\hline$\varepsilon=3$ & 1180 & $\begin{array}{c}\mathrm{q}_{\psi_{\text {Man" } \rightarrow \text { Man }}}=3 \\
\mathrm{q}_{\psi_{\text {Man' } \rightarrow \text { Man }}}\end{array}$ & 51 & 65 & $\begin{array}{l}\Phi_{\psi_{\text {Man" } \rightarrow \text { Man }}}=96 \\
\Phi_{\psi_{\text {Man' } \rightarrow \text { Man }}}=45\end{array}$ & 0.79 \\
\hline$\varepsilon=80$ & 2210 & $\begin{array}{c}\mathrm{q}_{\psi_{\text {Man" } \rightarrow \text { Man }}}=4 \\
\mathrm{q}_{\psi_{\text {Man' } \rightarrow \text { Man }}}\end{array}$ & 119 & 170 & $\begin{array}{c}\Phi_{\psi_{\text {Man" } \rightarrow \text { Man }}}=18 \\
\Phi_{\psi_{\text {Man' } \rightarrow \text { Man }}}\end{array}$ & 0.70 \\
\hline
\end{tabular}

The same trends are observed for the trisaccharide. The partition function is lower than those encountered for other trisaccharides, ${ }^{12-15}$ with the exception of the sulfated ones ${ }^{13,15}$ and $\alpha$ sophorotriose. ${ }^{14}$ However, at $\varepsilon=80$ the partition function almost doubles its value, indicating a large increase in flexibility. When comparing the disaccharide and the trisaccharide, at $\varepsilon=3$ it is shown that the $\mathrm{q}_{\psi}$ for the disaccharide is the same as that of the Man" $\rightarrow$ Man' linkage of the trisaccharide (Table 3), whereas that of the other linkage are largely reduced. It has already been predicted that a trisaccharide should be less flexible than a disaccharide, ${ }^{30}$ and also that the 
linkage closer to the reducing end should be less flexible than the other one, ${ }^{12}$ a phenomenon shown to hold in most cases. ${ }^{13,15}$ On the other hand, at $\varepsilon=80$, both of the trisaccharide linkages show to behave similarly between themselves and with that of the disaccharide (Table 3). A similar effect is shown to occur by measuring absolute flexibilities $(\Phi)$ : the flexibility increases at $\varepsilon=80$. The determination of individual absolute flexibilities for each linkage $\left(\Phi_{\psi}\right)$ show again that at $\varepsilon=3$, the Man" $\rightarrow$ Man' linkage behaves like that of the disaccharide, whereas the Man' $\rightarrow$ Man linkage shows a reduced flexibility (Table 3). On the other hand, at $\varepsilon=80$ no such effect was found to occur. Furthermore, the flexibilities increase with respect to those of the disaccharide, an effect originated in the low potential barriers found for the $\mathbf{A A} \leftrightarrow \mathbf{A B}$ and $\mathbf{A A} \leftrightarrow \mathbf{B A}$ interconversions (Table 3).

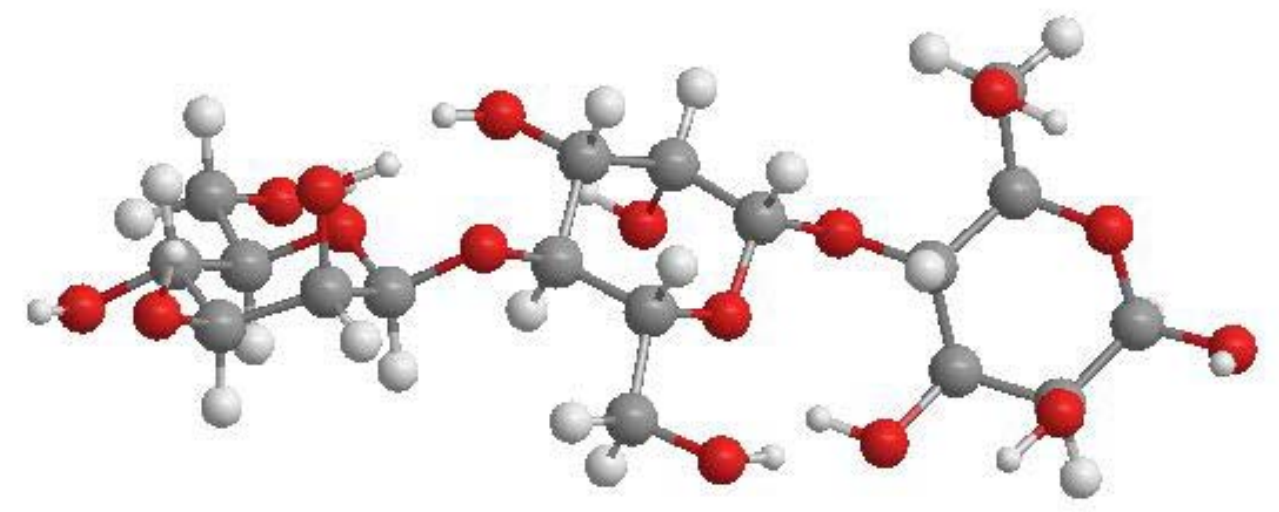

Figure 4. Molecular drawing for the minimum energy conformation calculated by MM3 ( $\varepsilon=3$ ) for $\beta$-mannotriose.

This work shows once more the advantages of fully relaxed calculations and mapping of trisaccharides by the way of 3D contours complementing those of the single disaccharides. The advent of better functional forms or variations in the parameterization in the future may lead to a better coincidence with experimental data by using the same approach.

\section{Methods}

The molecular mechanics program MM3 (92) (QCPE, Indiana University, USA), developed by Allinger and coworkers was used, ${ }^{8,9}$ modified as the MM3 (2000) version in the O-C-C-O and $\mathrm{O}-\mathrm{C}-\mathrm{O}-\mathrm{H}$ torsional parameters, $\mathrm{O}-\mathrm{H}$ hydrogen bonding parameters, and $\mathrm{C}-\mathrm{H}$ electronegativity correction. ${ }^{31}$ Besides, a suggested modification of the MM3 routines ${ }^{32}$ was made, by changing the maximum atomic movement from 0.25 to $0.10 \AA$. The dielectric constant was set as stated in the Results Section. Minimization was carried out by the block diagonal Newton-Raphson procedure for grid points and by the full-matrix procedure for minima, and was terminated using the default MM3 values. The dihedrals $\phi$ and $\psi$ for $\beta$-mannobiose are defined by atoms O5'-C1'- 
O1'-C4 and C5-C4-O1 '-C1', respectively, while $\phi^{\mathrm{H}}$ and $\psi^{\mathrm{H}}$ are defined by atoms $\mathrm{H} 1$ '-C1'-O1'$\mathrm{C} 4$ and $\mathrm{H} 4-\mathrm{C} 4-\mathrm{O} 1$ ' $-\mathrm{C} 1$ ', respectively. For the trisaccharide, the constituting monosaccharides are labeled as shown in Str. Block 1. The dihedral angles $\phi_{\text {Man' } \rightarrow \text { Man }}$ and $\psi_{\text {Man' } \rightarrow \text { Man }}$, and their hydrogen-related counterparts are defined exactly as those of the disaccharides, whereas those for the Man" $\rightarrow$ Man' linkage follow the same conventions, but nonprimed atoms should be primed, and those primed should be double-primed. The orientation of the hydroxyl hydrogen atoms is indicated by $\chi_{n}$, defined by the atoms $\mathrm{Hn}-\mathrm{Cn}-\mathrm{On}-\mathrm{H}(\mathrm{On})$, while $\chi_{6}$ is defined by the atoms C5-C6-O6-H(O6), and $\omega$ by the atoms O5-C5-C6-O6, singly- or double-primed when necessary. Their values are described by a one-letter code: ${ }^{33} \mathbf{S}$ for angles between -30 and + $30^{\circ}, \mathbf{g}$ for $30-90^{\circ}, \mathbf{T}$ for angles with absolute value larger than $150^{\circ}$, and $\mathbf{G}$ for angles between $30^{\circ}$ and $-90^{\circ}$. Free energies were calculated from the vibrational analysis of the minima, with no special treatment for the low-frequency vibrations: ${ }^{34}$ i.e. the effect of frequencies equal or lower than $20 \mathrm{~cm}^{-1}$ was added to the MM3 output values of vibrational enthalpies and entropies.

\section{Generation of disaccharide potential energy surfaces}

The maps were generated following the general procedures already described.3,10-15 Low energy conformers in different $\psi$ regions were chosen, with varied orientations of the hydroxyl and hydroxymethyl groups. These starting conformers were selected based on a previous study on cellobiose, ${ }^{12}$ but applying an iterative method in order to correct for the different configurations on $\mathrm{C} 2$ of both disaccharides. In this iterative procedure, ${ }^{3}$ the effect of rotations of alternate pairs of hydroxyl and hydroxymethyl groups were checked in order to find those of lower energy. The procedure was handled independently at $\varepsilon=3$ and $\varepsilon=80$. The $2 \mathrm{D}$ plots were obtained as described:10 beginning with all starting conformers in the A-B region (19 at $\varepsilon=3$ and 87 at $\varepsilon=80$ ), minimizations with a restrained $\psi$ in $10^{\circ}$ intervals were carried out both by means of the dihedral drivers 2 (sequential minimization) and 4 (minimization from the starting point), allowing the remaining variables to relax. The energy for each $\psi$ point was considered to be the lowest of all the calculations, thus giving rise to a $2 \mathrm{D}$ conformational adiabatic plot as a function of the $\psi$ angle (Fig. 1). A similar procedure was used to generate the $3 \mathrm{D} \phi, \psi$ maps, but extending on a $15^{\circ}$ grid at both the angles $\phi$ and $\psi$. In order to cover the main low-energy area, the angle $\psi$ was varied from $140^{\circ}$ to $305^{\circ}$, whereas $\phi$ was varied from $-40^{\circ}$ to $-160^{\circ}$. By recording only the lowest energy values for each $\phi, \psi$ combination, the conformational adiabatic maps, or energy surfaces as function of $\phi$ and $\psi$ angles were produced (Figure 2). A separate calculation for the remaining minima (B', C and D) was carried out with all the A-B starting structures.

\section{Generation of trisaccharide minima}

The general procedure was applied:12-15 different starting orientations of the hydroxyl and hydroxymethyl groups were tested, using the conformers that yielded at least one low-energy grid point in the disaccharide maps: for the reducing and non-reducing ends (Man and Man"), the orientations obtained for the reducing and non-reducing ends of the equivalent disaccharides were used, respectively. For Man', both the orientations occurring at the reducing and non- 
reducing end of the disaccharide were used. In this way, at $\varepsilon=3,96$ different orientations of the exocyclic groups were tested (four for Man and Man", and six for Man'), whereas at $\varepsilon=80,150$ different orientations appeared $(5 \times 6 \times 5)$. In an automated fashion, unrestrained MM3 fullmatrix calculations were carried out for points starting at each of those orientations, each one at a different combination of the $\phi, \psi$ angles (in either of both linkages) which gives rise to a major minimum in the disaccharide maps (A and B). Thus, 384 structures were tested $(96 \times 2 \times 2)$ at $\varepsilon=3$, and 600 at $\varepsilon=80$. The output was cleaned up for repetitions, structures with negative or zero eigenvalues, etc., and finally for each minimum, only those orientations with an energy up to 1 kcal above the lowest in that $\psi_{\text {Man' } \rightarrow \text { Man }}, \psi_{\text {Man" } \rightarrow \text { Man }}$, region (or higher than that value, but with a very low free energy) were left. In this way, at $\varepsilon=3,28$ different conformers were found, corresponding to 24 unique orientations of the exocyclic groups. On the other hand, at $\varepsilon=80,48$ different conformers were generated, all of them with unique orientations of the exocyclic groups.

\section{Generation of trisaccharide maps ${ }^{12-15}$}

The maps were generated as already described for the disaccharides. Both the dihedral drivers 2

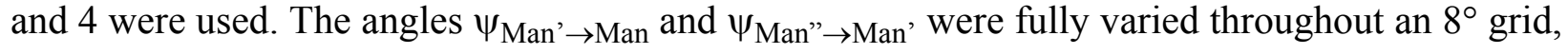
from $160^{\circ}$ to $280^{\circ}$. At each point, energies were calculated after minimization with restraints for these two angles but allowing the other variables (including the $\phi$ angles) to relax, starting from all the minima in the four regions generated as explained in the previous paragraph. The conformational adiabatic maps, or energy surfaces as function of both $\psi$ angles were produced (Figure 3).

Flexibility measurements. The absolute flexibilities for di- and trisaccharides were calculated as described previously. ${ }^{12,28,29}$ First, the energies and geometries of the transition states between minimum energy regions were calculated: they were first estimated analyzing the path from one minimum to another one in the adiabatic maps, and then determined by a full-matrix analysis, confirming that only one negative eigenvalue appeared. Then, the absolute flexibility $\Phi$ was calculated as:

$$
\Phi_{p \theta_{l}}=\sum_{i=1}^{n}\left[\left(\frac{e^{-E_{i} / R T}}{\sum_{k=1}^{n} e^{-E_{k} / R T}}\right) \times \sum_{j=1}^{m}\left(e^{-\left(E_{j}-E_{g m}\right) / R T}\right) \times\left(\frac{\sum_{l=1}^{p} \theta l, i-\theta l, j}{p \times 360^{\circ}}\right)\right]
$$

where $E_{g m}$ is the energy of the global minimum, $n$ is the number of minima (indexes $i$ and $k$ ), $m$ the number of transition states (index $j$ ) surrounding minimum $i, p$ is the number of dihedral angles $\theta$ been monitored (index $l$ ), measured in degrees, $\mathrm{R}$ is the universal gas constant, and $\mathrm{T}$ 
the temperature (set to $25{ }^{\circ} \mathrm{C}=298.16 \mathrm{~K}$ ). The above-mentioned formula was also utilized to calculate the absolute flexibilities of the trisaccharides with respect to each glycosidic linkage. For this purpose, the absolute flexibility for each of the paths involving a neat variation of the $\psi$ angle corresponding to this linkage was calculated independently. For each $\psi$ angle all these flexibilities, weighed through their Boltzmann populations were added. The partition functions were calculated as: ${ }^{11,12}$

$$
q_{p \theta_{l}}=\prod_{l=1}^{p} \Delta \theta_{l} \times \sum_{i=1}^{E S} e^{-\left(E_{i}-E_{g m}\right) / R T}
$$

where $\Delta \theta$ are the grid spacings $\left(10^{\circ}\right.$ for the disaccharide $2 \mathrm{D}$ maps, $15^{\circ}$ for the disaccharide $3 \mathrm{D}$ maps, $8^{\circ}$ for the trisaccharide maps) and the summation is carried out over the entire surface determined. The partial flexibility on each of the $\psi$ torsional angles of the trisaccharides, was

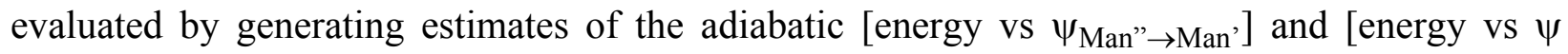
Man' $\rightarrow$ Man] relationships. These were created by recording, the minimum energy in the maps for each column and for each row of data, respectively. From these data, partial $\mathrm{q}_{\psi_{\text {Man" } \rightarrow \text { Man }}}$, and $\mathrm{q}_{\psi}$ Man' $\rightarrow$ Man values were calculated with the above-mentioned equation.

\section{Acknowledgements}

The author is Research Member of the National Research Council of Argentina (CONICET). This work was supported by a grant from the University of Buenos Aires (X-174).

\section{References and Footnotes}

*MM3 Potential energy surfaces of trisaccharides. Part 5.

1. French, A. D.; Brady, J. W. ACS Symp. Ser. 1989, 430, 1.

2. Tran, V.; Buléon, A.; Imberty, A.; Pérez, S. Biopolymers 1989, 28, 679.

3. Stortz, C. A. Carbohydr. Res. 1999, 322, 77.

4. Dowd, M. K.; Zeng, J.; French, A. D.; Reilly, P. J. Carbohydr. Res. 1992, 230, 223.

5. Dowd, M. K.; French, A. D.; Reilly, P. J. Carbohydr. Res. 1992, 233, 15.

6. Dowd, M. K.; French, A. D.; Reilly, P. J. J. Carbohydr. Chem. 1995, 14, 589.

7. Mendonca, S.; Johnson, G. P.; French, A. D.; Laine, R. A. J. Phys. Chem. A 2002, 106, 4115.

8. Allinger, N. L.; Yuh, Y. H.; Lii, J-H. J. Am. Chem. Soc. 1989, 111, 8551.

9. Allinger, N. L.; Rahman, M.; Lii, J-H. J. Am. Chem. Soc. 1990, 112, 8293. 
10. Stortz, C. A.; Cerezo, A. S. Carbohydr. Res., 2002, 337, 1861.

11. Stortz, C. A.; Cerezo, A. S. J. Carbohydr. Chem. 2003, 22, 217.

12. Stortz, C. A.; Cerezo, A. S. Carbohydr. Res., 2003, 338, 95.

13. Stortz, C. A.; Cerezo, A. S. Biopolymers 2003, 70, 227.

14. Stortz, C. A.; Cerezo, A. S. Carbohydr. Res. 2003, 338, 1679.

15. Stortz, C. A. Carbohydr. Res. 2004, 339, 2381.

16. Stephen, A. M. In The Polysaccharides; Aspinall, G. O., Ed.; Academic Press Inc.: Orlando, USA, 1983; Vol. 2, pp 97-193.

17. Tvaroska, I.; Perez, S.;Noble, O.; Taravel, F. Biopolymers 1987, $26,1499$.

18. Jiménez-Barbero, J.; Noble, O.; Pfeffer, C.; Perez, S. New J. Chem. 1988, 12, 941.

19. Bergamini, J. F.; Boisset, C.; Mazeau, K.; Heyraud, A.; Taravel, F. R. New J. Chem. 1995, 19, 115.

20. Petkowicz, C. L. O.; Reicher, F.; Mazeau, K. Carbohydr. Polymers 1998, 37, 25.

21. Sheldrick, B.; Mackie, W.; Akrigg, D. Carbohydr. Res. 1984, 132, 1.

22. Mackie, W.; Sheldrick, B.; Akrigg, D.; Pérez, S. Int. J. Biol. Macromol. 1986, 8, 43.

23. Cappelletti, C. I.; Navarro, D. A.; Manzi, A. E.; Stortz, C. A.; Cerezo, A. S. ARKIVOC submitted.

24. Chanzy, H.; Pérez, S.; Miller, D. P.; Paradosi, G.; Winter, W. T. Macromolecules 1987, 20, 2407.

25. Atkins, E. D. T.; Farnell, S.; Mackie, W.; Sheldrick, B. Biopolymers 1988, 27, 1097.

26. Stortz, C. A.; Cerezo, A. S. J. Carbohydr. Chem. 2002, 21, 355.

27. French, A. D.; Kelterer, A-M.; Johnson, G. P.; Dowd, M. K.; Cramer, C. J. J. Comput. Chem. 2001, 22, 65.

28. Koča, J. J. Mol. Struct. 1993, 291, 255.

29. Koča, J.; Pérez, S.; Imberty, A. J. Comput. Chem. 1995, 16, 296.

30. Mazeau, K.; Pérez, S. Carbohydr. Res. 1998, 311, 203.

31. Stortz, C. A. J. Comput. Chem. 2005, 26, 471.

32. MM3 (96). Bull. QCPE 1997, 17 (1), 3.

33. Engelsen, S. B.; Koča, J.; Braccini, I.; Hervé du Penhoat, C.; Pérez, S. Carbohydr. Res. 1995, 276, 1.

34. Engelsen, S. B.; Rasmussen, K. J. Carbohydr. Chem. 1997, 16, 773. 